\title{
Renovar contenidos docentes: organización de la producción/dirección de operaciones
}

\author{
Bringing up to date teaching contents: production/operations management
}

\author{
Albert Corominas \\ Instituto de Organización y Control de Sistemas Industriales, Departamento de Organización de Empresas, ETSEIB. Universitat \\ Politècnica de Catalunya, Avda Diagonal 647, Barcelona \\ albert.corominas@upc.edu
}

Fecha de recepción: 24-10-08

Fecha de aceptación: 03-03-10

Resumen: Se justifica la necesidad de replantear el paradigma docente, con la repercusión consiguiente en la investigación, en el ámbito de la organización de la producción / dirección de operaciones, como consecuencia de los cambios en los sistemas productivos y su entorno y en el acervo de conocimientos y de instrumentos disponibles. En relación con estos cambios, se discute el enfoque de algunos temas característicos de la disciplina. Se resalta la importancia, al respecto, del fomento de propuestas críticas e innovadoras en los proyectos académicos correspondientes a concursos de plazas de profesorado.

Palabras clave: organización de la producción, dirección de operaciones, ingeniería de organización, docencia.

Abstract: The need to reconsider for the teaching paradigm currently in force in the field of production / operations management, with the corresponding impact on research, is justified because of the changes in production systems and their environment and in the available knowledge and tools. Concerning these changes, the approach of some topics characteristics of the discipline is discussed. The importance of fostering critical and innovative proposals in the selection processes for filling vacancies in the academic staff of universities is stressed.

Key words: production / operations management, industrial engineering, teaching

\section{Introducción: por qué hay que plantear la renovación de los programas docentes relativos a la dirección de operaciones}

Lo que puede llamarse paradigma vigente en la docencia de la organización de la producción (para la que también se utiliza la expresión «dirección de operaciones», aunque, estrictamente, ésta tiene un alcance más limitado) se estableció, en pocos años, en los inicios de la segunda mitad del siglo pasado. Ahora, pues, de casi todo hace ya más o menos cincuenta años (Holt et al., 1955; Holt et al., 1956; la dinámica de sistemas, 1957; el PERT, 1958; el GSP (General Simulation Program) de K. D. Tocher, 1958; Hopp, 2004; Chopra et al., 2004; Singhal y Singhal, 2007), cuando no más de cien o casi cien (FordT: 1908; Harris, 1913; F.W. Taylor muere en 1915) y parece por ello obligado que quienes nos dedicamos a la docencia y la investigación en el ámbito de la organización de la producción o la dirección de operaciones (Alfalla y Domínguez, 2002), con independencia de cómo denomine cada cual a esta disciplina (nos referiremos a ella en lo sucesivo como OP/DO), nos detengamos a reflexionar si estamos enseñando lo que deberíamos.

Últimamente, de unos años a esta parte, a propósito de la adaptación del sistema español al Espacio Europeo de Educación Superior (EEES), se habla mucho de renovación de la docencia universitaria, por lo que respecta a la metodología docente. Pero también se asocia el EEES con la necesidad de definir los objetivos de los planes de estudios y de las asignaturas, lo que debe implicar, en la mayoría de los casos, una revisión de los contenidos.

Por consiguiente, el EEES implica un proceso de renovación, que constituye una oportunidad de mejora.

No obstante, lo más relevante para el caso que nos ocupa es el hecho de que los conceptos básicos y los temarios, en lo que a la OP/DO se refiere, se forjaron hace ya más de cincuenta años y cristalizaron en el primer libro de texto de Ellwood S. Buffa (Buffa, I96I), cuyo papel crucial en nuestro ámbito ha sido recordado recientemente (Singhal et al., 2007), 
con motivo del fallecimiento de su autor. En Hayes (2008) se indica que la disciplina está sometida a tensión y se pregunta por su futuro.

En cincuenta años, el mundo ha cambiado, las universidades y las empresas han generado una cantidad inmensa de nuevos conocimientos (no debemos olvidar que, este ámbito, a diferencia de lo que ocurre en algunos otros, una gran parte de los conocimientos nuevos procede de las empresas) y las herramientas disponibles para el cálculo superan en muchos órdenes de magnitud a las de hace medio siglo, lo que permite resolver problemas que en aquel entonces estaban completamente fuera del alcance incluso de los equipos más avanzados

Es decir, hay que renovar o, al menos, hay que reflexionar sobre la necesidad de renovar la docencia de la OP/DO.Y el elemento importante y específico de esta renovación es la del contenido, ya que la renovación pedagógica, también necesaria, se producirá como parte de un proceso que afectará a toda la enseñanza universitaria. Recientemente el profesor James P.Walsh' nos ha recordado que Murray Davis aconsejaba (Davis, 197I) poner patas arriba nuestros supuestos si queríamos encontrar cuestiones interesantes.

El objetivo de este trabajo consiste en proporcionar elementos para la reflexión y el debate sobre la renovación de los contenidos de la OP/DO. No se pretende proponer aquí alternativas globales al paradigma vigente, ya que ésta es una tarea que requiere la conjunción de esfuerzos numerosos. No es una propuesta iconoclasta, como la de algunos impulsores de nuevos paradigmas de gestión, que suelen incitar a que se eche por la borda, por nocivo y obsoleto, todo conocimiento del pasado; nuestra disciplina ha acumulado un acervo muy valioso del que sigue vigente una muy buena parte de sus elementos, aunque a veces a condición de insertarlos en un esquema actualizado. Por supuesto, la crítica explícita o implícita en el texto es aplicable asimismo a una parte de la actividad docente de propio autor.

El resto del artículo está organizado como sigue. La sección siguiente se refiere a las causas de que los programas docentes no se hayan renovado suficientemente; en la sección 3 trata de los cambios significativos para la OP/DO acontecidos, aproximadamente, en los últimos cincuenta años y, en la siguiente, se analizan críticamente los enfoques tradicionales de diversos temas importantes en el ámbito de la OP/DO. El trabajo finaliza con unas breves conclusiones.

\section{Sobre las causas de la actualización insuficiente de los programas}

Dice Stigler, en uno de sus ensayos (Stigler, 1978), a propósito de una ley económica no verificada empíricamente, prescindible y no utilizada, pese a lo cual figura persistentemente en decenas de libros de texto, que «los manuales de una disciplina desempeñan un poderoso papel conservador en la transmisión de la doctrina». Efectivamente, en general, los nuevos libros de texto se apoyan en los anteriores y difícilmente suprimen un tema, sin perjuicio de que reduzcan la extensión que le asignan hasta convertir la explicación en algo incomprensible o simplemente inútil. $O$ incluso equivocado: sería interesante estudiar dónde se inició la propagación del error según el cual el punto que minimiza la suma ponderada de distancias a otros dados es el centro de gravedad de los mismos (Chase et al., 2005; Heizer y Render, 2007a; Kuo y White, 2004). Por otra parte, es conocido que muchas personas mantienen, ante los libros de texto, una actitud reverencial, que las inclina a creer sin reservas las afirmaciones que contienen, e incluso a resistirse a aceptar la falsedad de las mismas cuando ésta se pone de manifiesto.

En Taj et al. (1996) se concluye que los programas no cambian al mismo ritmo que la realidad y no reflejan los resultados de la investigación del propio profesorado que imparte las enseñanzas.

Se ha dicho que esta resistencia al cambio podría ser debida a la actitud acomodaticia que lleva a enseñar lo que ya sabemos, que sería lo que nos enseñaron cuando éramos estudiantes. Pero esta explicación es manifiestamente insuficiente, por cuanto no da cuenta de por qué quienes investigan no incorporan el resultado de sus investigaciones a la enseñanza. No se trata de los aspectos técnicamente más complejos, que pueden no tener cabida si no es en el doctorado, sino de los conceptos y los enfoques.

En el caso español, uno de los motivos principales de la falta de renovación de los contenidos de los programas han sido, al menos hasta ahora, los sistemas de selección del profesorado (en cambio, al res-

(http://meeting.aomonline.org/2008/index.php?option=com_content\&task=view\&id= I \&ltemid= I, consultada el 28/I I/2007) 
pecto apenas han tenido impacto alguno las denostadas directrices ministeriales que, se decía, encorsetaban la libre configuración de los programas de las asignaturas). Las memorias que presentan quienes concursan a una plaza (o, estos últimos tiempos y hasta hace poco, a una habilitación), por lo menos en el área de Organización de Empresas, son casi siempre documentos estereotipados, en los que se elude el riesgo, por la vía de soslayar cualquier juicio sobre las diversas escuelas o teorías (enumeradas y sucintamente descritas) y por la de reproducir, con muy pocas variaciones, si las hay, los programas propuestos en su momento por quienes les precedieron. Ello puede considerarse explicable, normal y lógico, pero debería evitarse porque conduce a una progresiva fosilización de los contenidos. Y sólo puede evitarse con un cambio radical de criterios en virtud del cual se premie la innovación y no el conformismo.

Una de las facetas del economista es la de predicador, dice también Stigler (Stigler, 1978). Y también se predica en el ámbito de la OP/DO: reingeniería y mejora continúa, innovación. Otra cosa es hasta qué punto se aplica en nuestro ámbito lo que en él se propone.

\section{3. ¿Qué ha cambiado significativamente en los últimos cincuenta años?}

Hemos asistido, en el último medio siglo, a cambios substanciales en la realidad de los sistemas productivos y en la forma de concebirlos y estudiarlos. En lo que sigue se describen brevemente, en un orden que no corresponde necesariamente a su importancia, los que el autor considera más relevantes en relación con los contenidos de la enseñanza en OP/DO.

En primer lugar, como consecuencia de la evolución de los sistemas de aprovisionamiento-produccióndistribución, se ha pasado de una concepción de los sistemas productivos como unidades conectadas, por un lado, con los proveedores y, por otro, con los clientes, y pasando por los «sistemas productivos y logísticos», a la aceptación general del término «cadena de suministro» (que, por cierto, convendría sustituir por «red de suministro», para que el significado literal del término se ajustara mejor al concepto asociado.

En segundo lugar, la innovación en toda clase de procesos y productos, incluyendo los bienes de equipo para fábricas y oficinas, se ha acelerado y generaliza- do. A diferencia de lo que ocurría en el pasado, incluso productos que podrían calificarse como maduros, mejoran constantemente y ofrecen nuevas prestaciones, a veces acompañadas de una reducción de su precio.

En tercer lugar, en los denominados países industrializados, el predominio de la manufactura en la composición del empleo y de la producción ha dado paso al de los servicios, ampliamente mayoritarios desde hace ya muchos años, tanto en uno como en otro de los criterios citados (Karmarkar, 2004; Machuca et al., 2007; Smith et al., 2007). Paralelamente, la frontera entre bienes y servicios se ha ido difuminando y cada vez es menos habitual que un producto sea puramente un bien o puramente un servicio.

En cuarto lugar, algunas empresas han generado nuevos enfoques y nuevas técnicas, al margen del paradigma académico vigente. El denominado sistema de producción de Toyota (Monden, 1983) o producción ajustada (lean production; Womack et al., 1990) tiene un encaje difícil en los esquemas tradicionales.

En quinto lugar, el deterioro real, y acelerado, del medio ambiente y la creciente conciencia del mismo han dado un empuje vigoroso al concepto de logística inversa, en el sentido más amplio de actividades relativas a la recogida y eliminación de residuos junto con las de reutilización, reciclaje y remanufactura (Bañegil y Rubio, 2005).

En sexto lugar, el enfoque monocriterio ha dado paso al multicriterio. Éste tiene orígenes diversos que se remontan al menos a Benjamin Franklin, pero en el ámbito académico no adquiere una fuerte presencia hasta en los años setentas del siglo pasado. De hecho, el grupo EWG-MCDA (EURO Working Group Multicriteria Decision Aiding) se constituyó en Bruselas en 1975, con ocasión del EURO I (es decir, el primer congreso europeo de investigación operativa).

En séptimo lugar y finalmente, la evolución de los algoritmos, el hardware, el software y las redes de comunicaciones permite disponer de instrumentos para la optimización y la simulación que son millones de veces más eficientes que los de hace cincuenta años (ver, por ejemplo, Bixby, 2002). Este aumento de muchos órdenes de magnitud en la eficiencia de dichas herramientas implica un cambio radical en lo que respecta al enfoque de los problemas, en las dimensiones y en la complejidad de los que ahora pueden ser tratados y antes resultaban inabordables salvo de forma rudimentaria. 


\section{4. ¿Y qué no ha cambiado, ha cambiado insuficientemente o no ha cambiado en la dirección adecuada?}

Sin pretensión de exhaustividad, analizamos algunos temas característicos de la disciplina o cuestiones relacionadas con la misma para llegar a orientaciones sobre cómo, a nuestro entender, deberían enfocarse.

\section{Estrategia y táctica}

Muchos problemas de nivel táctico han podido ser abordados eficientemente mediante técnicas cuantitativas, incluso en las fases iniciales de desarrollo de las mismas, pero no las decisiones estratégicas que, en consecuencia, han tenido poco peso en los programas de las asignaturas en que ha predominado el enfoque cuantitativo. Los contenidos deberían reequilibrarse en este sentido, máxime cuando la capacidad de las técnicas cuantitativas para tratar problemas estratégicos ha aumentado significativamente en los últimos años. Esta mayor capacidad de las técnicas permite también en algunos casos una mayor integración, que puede denominarse vertical, de las decisiones estratégicas y las tácticas y superar así algunas limitaciones del clásico enfoque jerárquico.

\section{Manufactura y servicios}

Generalmente, los programas docentes y los libros de texto se desarrollan sobre un esquema basado en la manufactura y, de vez en cuando, incluyen unos comentarios sobre las diferencias que presentan los servicios en relación con la manufactura. Pero, como se ha indicado anteriormente, este enfoque no parece adecuado cuando los servicios son mayoritarios y los bienes y los servicios se presentan, frecuentemente, entreverados.

Por otra parte, es difícil tratar sobre un objeto de límites difusos y esto es lo que ocurre con los servicios, que se suelen definir por exclusión (todas las actividades productivas con excepción de manufactura, construcción, minería, agricultura, ganadería, caza y pesca), de lo que resulta un conjunto heterogéneo, a cuyos elementos difícilmente se les puede aplicar los mismos enfoques y las mismas técnicas. Al respecto, el enfoque propuesto en Sampson y Froehle (2006) merece ser considerado atentamente; algunas de las ideas contenidas en dicho trabajo son: que en una empresa tiene lugar una variedad de procesos, que algunos de estos procesos son procesos de servicios, que se caracterizan porque en ellos el cliente aporta una parte significativa de los inputs. Este enfoque puede ser un buen punto de partida para superar la hasta ahora desequilibrada dicotomía manufactura/servicios.

\section{Localización de instalaciones}

Aunque, como se ha indicado más arriba, el uso del término «cadena de suministro» se ha generalizado, sigue predominando el tratamiento del problema de localización como la de elementos aislados y no como la determinación de la estructura de una red. Incluso en textos avanzados el tratamiento no va más allá de exponer el modelo de Balinski (Balinski, 1965), que procede de una época en que ni siquiera se había acuñado el término «cadena de suministro». Los modelos de localización de elementos aislados siguen siendo útiles conceptualmente y desde el punto de vista didáctico, pero a estas alturas tendrían que ir acompañados de modelos, aunque sean esquemáticos, para determinar la configuración de una cadena de suministro. Para esto no sirve la regla del centro de gravedad, aunque no fuera falsa.

\section{Logística inversa}

En muchos programas y libros de texto (de hecho, en la inmensa mayoría) simplemente no aparece este concepto, pese a sus importantes implicaciones en el diseño y la gestión de la cadena de suministro (por ejemplo, en la planificación agregada y en la gestión de inventarios). Una excepción interesante es Miranda et al. (2005), pero, pese a que algunos de sus autores son especialistas en el tema (Rubio et al., 2008), se le dedica un espacio muy reducido.

La importancia presente y futura de la logística inversa debería reflejarse en los programas docentes, tanto en lo que se refiere a la recolección del producto usado y su tratamiento como residuo, como en lo relativo a cadenas de suministro en bucle cerrado (por reinserción en la cadena de aprovisionamiento de productos, componentes o materiales) y los nuevos problemas que esta supone (básicamente, por la mayor incidencia de magnitudes aleatorias).

\section{Métodos y tiempos, descripción, diseño y mejora de métodos}

Los tradicionales «métodos y tiempos», de regusto taylorista, siguen siendo útiles, aunque han desapa- 
recido de algunos programas porque alguien los debe de haber considerado vetustos (y difíciles de encajar en el paradigma basado en modelos matemáticos). Quienes así piensan tienen, desde luego, parte de razón: los procesos manuales y de alcance limitado que aparecen en las presentaciones tradicionales de estudio y mejora de métodos tienen cada vez menor peso en los procesos productivos y logísticos y la mejora de los mismos no es en muchos casos la clave de la competitividad. Aun así, el famoso y celebrado SMED (Single Minute Exchange of Die) no deja de ser una aplicación de los métodos y tiempos. La mejora de métodos no es suficiente, pero sigue siendo necesaria y la opción adecuada no es, en cuanto a la docencia, eliminarla del temario, sino convertirla en la descripción y mejora de procesos en un sentido amplio.

\section{Renovación de equipos}

Los modelos de renovación de equipos únicos, en un contexto determinista, sin progreso tecnológico y con horizonte ilimitado se resuelven elegante y fácilmente. Pero, iqué nos dicen sobre lo que tenemos que hacer en la realidad? Nunca ha sido especialmente difícil plantear modelos (por ejemplo, basados en grafos) de renovación de equipos múltiples, con horizonte limitado (aunque puede ser bastante dilatado) y con progreso tecnológico. Desde hace tiempo, tales modelos, aun los de grandes dimensiones, se pueden resolver con poco tiempo de cálculo. ¿No debería invertirse, por consiguiente, el esquema típico de presentación del problema de renovación en contexto determinista? De hecho, es sorprendente la ausencia de los modelos de renovación en muchos libros de texto, más aún cuando algunos dedican una cierta atención a la fiabilidad, lo cual proporciona los conceptos necesarios para abordar los modelos de renovación de elementos que se averían aleatoriamente y el mantenimiento preventivo.

\section{Planificación agregada}

El concepto de PA se remonta, al menos, a Rathenau (Rathenau, 1918, The New Economy; citado en Urwich y Brecht, 1984) y se trata en manuales profesionales tan antiguos como (Alford y Bangs, 1944). Pero ha ido perdiendo peso desde hace algún tiempo en libros de texto y en los manuales (Boiteux et al., 2007).

En un libro de texto muy difundido y que nos parece representativo (Heizer y Render, 2007b) se defi- ne como «un método para determinar la cantidad de producción y su desarrollo en el tiempo para un plazo medio».

Por otra parte, la definición citada no concuerda con las de autores que consideran la PA desde un punto de vista más integrador de las áreas funcionales de la empresa. Obsérvese que la correcta insistencia en la necesaria coordinación de todos los elementos de la cadena de suministro y en los beneficios que de ella pueden derivarse no tiene correlación en un discurso análogo sobre la necesidad de coordinar las áreas funcionales de la empresa.

De hecho, el concepto clave para coordinar las actividades de las áreas funcionales es justamente la PA. En Singhal y Singhal (2007) se dice que «la atención en las cuestiones relativas a la PA de la producción trajo al primer plano el papel central de la OP/DO en la conexión de otras funciones como contabilidad, finanzas, gestión de recursos humanos, sistemas de información, marketing y estrategia».Y en el mismo trabajo, dedicado a la aportación pionera de Holt, Modigliani, Muth y Simon (Holt et al., 1955; Holt et al., 1956), se dice que el problema abordado por estos autores «juega un papel fundamental en la integración de las funciones de OP/DO, marketing y finanzas». En Singhal et al. (2007), un trabajo dedicado a las aportaciones de E.S. Buffa, se afirma que «las decisiones de planificación agregada de la producción juegan un papel clave en la planificación de recursos del conjunto de la organización y en la integración de la organización, al conectar operaciones con contabilidad, distribución, finanzas, gestión de recursos humanos y marketing».

¿Cómo se presenta habitualmente la PA? De entrada, como la planificación agregada «de la producción». Obsérvese que así se la denomina en Singhal y Singhal (2007) y en Singhal et al. (2007), pese al papel integrador que se le atribuye. Se supone entonces que la PA tiene como entrada una previsión de demanda, establecida por el área comercial, y que proporciona como resultado un plan de producción, con decisiones asociadas relativas a la plantilla (horas extras, contrataciones y despidos); dicho resultado se comunica a finanzas y a compras para que, de acuerdo con el mismo, tomen las decisiones pertinentes. No puede decirse con propiedad, por consiguiente, que la PA integre las decisiones de las diversas áreas funcionales, puesto que las únicas decisiones explícitas en el proceso son las de producción y algunas de las de personal; las otras áreas actúan como proveedoras o receptoras de datos. Desde luego, el proceso de elaborar un plan agre- 
gado favorece la integración, porque propicia los contactos entre áreas y este proceso puede llevar a revisar el plan de demanda o tal vez a que finanzas, por ejemplo, detecte que el resultado del plan no es viable desde su punto de vista y ello obligue a reconsiderarlo.

Pero, en cualquier caso, este enfoque no conduce sino a un conjunto de decisiones subóptimas. Para obtener un plan óptimo se debe enfocar como la PA de la empresa y no como la PA de la producción, es decir, las decisiones de unas áreas no se deben subordinar a las de otras, sino que deben ser consideradas simultáneamente, al mismo nivel. Por supuesto, hasta hace algunos años esto no resultaba viable técnicamente, pero el desarrollo de los instrumentos de cálculo lo hace ahora posible.

Este mismo desarrollo permite considerar una división del horizonte de planificación en períodos más breves (semanas, por ejemplo) que los que los textos consideran típicos (meses) y, en general, flexibiliza la necesidad de agregar productos y recursos y suaviza las fronteras entre los diversos planes de la jerarquía planificación estratégica / planificación táctica / planificación operativa / programación de actividades, es decir, permite incorporar en los modelos de uno de los niveles de esta jerarquía decisiones correspondientes a los niveles inmediatamente superior e inmediatamente inferior.

Así pues, pensamos que el concepto de PA debe englobar el área comercial, compras, personal, producción, finanzas y distribución.

Por otra parte, aunque algunas decisiones sobre la gestión del personal se han incluido tradicionalmente en los modelos que se proponen en los libros de texto, este aspecto de la PA requiere ser revisado a fondo, Por una parte, porque las modalidades de gestión flexible del tiempo de trabajo no se reducen a la utilización de horas extras. Por otra, porque los, digamos tradicionales, modelos del tipo hire \& fire, que parten de los supuestos de que se puede despedir y contratar sin restricciones y de que la eficiencia del personal recientemente contratado no difiere de la del personal con mayor antigüedad son, en muchos entornos, irrealistas $y$, en el nuestro, entre otros, socialmente inaceptables.

De donde deducimos que los modelos de PA han de ser capaces de tratar las diversas modalidades existentes de gestión del tiempo de trabajo y de tener en cuenta el aprendizaje.
Finalmente, la logística inversa debería incorporarse a los modelos que se han propuesto para la PA, en los que normalmente no figura.

\section{Gestión de inventarios}

El tratamiento de la gestión de inventarios adolece de la separación entre ítems con demanda independiente e ítems con demanda dependiente, que se suelen tratar en partes separadas de la asignatura.

Los primeros dan lugar a modelos matemáticos que, aunque derivan en última instancia de la fórmula del lote económico (Harris, 1913), han surgido en el ámbito de la investigación operativa y suelen tener un capítulo específico en los libros de introducción a dicha disciplina, en los que el objeto de estudio es el modelo matemático, no la gestión de inventarios en la realidad (como el título del capítulo suele ser «gestión de inventarios» o equivalente ello tiene una consecuencia muy negativa: produce una confusión entre la gestión, que implica el uso de algunos modelos pero que incluye también muchas otras facetas, y los modelos mismos).

La mencionada fórmula del lote económico (Harris, 1913), aunque corresponde al supuesto altamente improbable de demanda determinista uniforme, permite introducir conceptos básicos asociados a la gestión de inventarios y la propia fórmula permite estudiar la sensibilidad de los costes en relación con parámetros como el coste de lanzamiento y el de posesión. Ahora bien, en muchos textos o programas la discusión sobre la fórmula de Harris para la EOQ (Economic Order Quantity) le sigue la correspondiente a la EPQ (Economic Production Quantity), en la que se supone que tenemos una máquina que se utiliza para obtener un único producto, con demanda uniforme, y que la tasa de producción de la máquina es superior a la de demanda del producto, por lo que se tienen que alternar fases de producción y consumo con las de sólo consumo, de modo que al principio de cada fase de producción-consumo se incurre en un coste de lanzamiento. El estudio de este modelo tiene sentido como preparación al caso multiproducto, pero como éste es bastante más difícil no figura en los libros de texto menos avanzados (este es el caso, por ejemplo, de Heizer y Render, 2007b), con lo cual el estudio de la EPQ puede suscitar entonces preguntas de difícil respuesta: (i) ipor qué en un contexto determinista y con demanda uniforme en un horizonte temporal ilimitado tenemos un sistema productivo de capacidad superior a la demanda? y (ii) ipor qué hay un coste de lan- 
zamiento si la máquina está dedicada al único producto, por lo que no se requiere un cambio de herramientas o similar? (es cierto que el sistema puede requerir un calentamiento previo, por ejemplo, con el consiguiente consumo de energía, pero este supuesto u otros similares no son aplicables a la mayoría de situaciones reales, de lo que se deduce que se debería analizar el caso sin coste de lanzamiento, o con coste de lanzamiento despreciable, con la conclusión de que la máquina debería funcionar a ráfagas tan cortas como fuera técnicamente posible). De hecho, la conclusión más general debería ser la de que, en los supuestos del modelo, la máquina debería tener una tasa de producción igual a la tasa de demanda y que la producción no debería interrumpirse: sería un sistema just-in-time, con inventario cero.

El párrafo anterior trata de una cuestión que puede considerarse menor (la EPQ) pero pone de manifiesto que coexisten en muchos libros de texto elementos contradictorios, o no suficientemente engarzados, como la EPQ y el JIT. Los que en su momento han sido (o han sido considerados como) nuevos enfoques (el JIT, el OPT -caso insólito: un paquete informático de contenido secreto elevado a concepto básico en multitud de libros de texto-, etc.) se han incorporado progresivamente como incrustaciones, como cuerpos extraños, sin integrarse en el discurso general, ni siquiera en cuanto a la forma (un texto de enfoque moderadamente cuantitativo puede tener intercalado un capítulo totalmente cualitativo sobre el JIT).

Pero en relación con los modelos para ítems con demanda independiente se plantean otras cuestiones. Por ejemplo, ipuede soslayarse el efecto, aguas arriba, de las reglas para lanzar pedidos que se apliquen en el último nivel de la cadena de suministro?; es decir, ¿no se debe incluir un tratamiento aunque sea sucinto del efecto bullwhip (Forrester, 196I; Lee et al. 1997a, 1997b) incluso en cursos no avanzados sobre gestión de inventarios? $\bigcirc$ bien, ¿para qué se necesita a estas alturas un algoritmo heurístico para sustituir al propuesto en Wagner y Within (1958)?

\section{Programación de actividades}

Los problemas de programación de actividades (de scheduling, para precisar rápidamente a qué nos referimos) se suelen presentar a partir de modelos basados en supuestos extremadamente restrictivos, elaborados en los cincuentas (por ejemplo: Johnson, 1954) y sesentas del siglo pasado, cuando la teoría del scheduling daba los primeros pasos en un cami- no que la conduciría progresivamente a una especie de rama de la matemática discreta.

Sería razonable partir de una descripción de los entornos en que se presenta realmente el problema para, desde ella, y teniendo en cuenta las limitaciones de las técnicas disponibles, llegar a modelos tratables. Pero la realidad no suele asomar por parte alguna: las operaciones pueden esperar ilimitadamente, los buffers son infinitos, los medios de transporte están siempre disponibles y llevan a cabo su tarea en un tiempo insignificante, los trabajos no se pueden dividir ni agrupar, no es posible que un recurso (una máquina) opere simultáneamente sobre más de un trabajo ni que un trabajo sea tratado simultáneamente por más de una máquina, etc. En particular, en los problemas de máquinas en serie se suele suponer que, para una tarea cualquiera, sólo hay una máquina capaz de llevarla a cabo, lo cual, desde luego, en la realidad muchas veces no es cierto (Gao et al, 2007). . Claro está que también hay modelos para máquinas en paralelo, pero éstas sólo son capaces de realizar un solo tipo de tarea; además, tal vez por motivos históricos, raramente se trata sobre máquinas en paralelo salvo en cursos avanzados.

Sin olvidar (otra vez el JIT) que una buena parte de los criterios clásicos en scheduling responden a la idea de hacer las cosas cuanto antes y no a la de hacerlas justo a tiempo.

En este apartado cabe también una discusión sobre la programación de actividades correspondientes a proyectos singulares. Parece obligado preguntarse si hay que seguir haciendo énfasis en la representación de las precedencias mediante un grafo con las actividades en los arcos (digamos, tipo PERT), que presenta varios inconvenientes y que nadie utiliza para representar las precedencias en el ámbito de otros problemas como los de scheduling o la asignación de tareas en líneas de producción o de montaje, en los cuales se usa exclusivamente la representación con las actividades en los vértices. Otra herencias cuestionables del PERT son: (i) el famoso supuesto de que la duración de una actividad se ajusta a una ley beta (lo que, desde luego, no es cierto en todos los casos y habría que ver si lo es en alguno: Bowman, 2007; Mohan et al., 2007); (ii) la fórmula, incorrecta, para calcular la esperanza matemática de la distribución a partir de los valores pesimista, más probable y optimista y (iii) el supuesto de que las duraciones de las actividades, y en particular las del camino crítico, son independientes. Más importante aún, en nuestra opinión, es el tratamiento somero que, en el mejor de 
los casos, se dedica a la programación de actividades en proyectos cuando hay limitación de recursos (es decir, siempre, salvo, quizá, en especialísimos proyectos de tipo militar).

\section{$\mathrm{Y}$ algunos temas más}

Por supuesto, hay otras cuestiones importantes. Por ejemplo, la escasa presencia del enfoque multicriterio, tanto desde un punto de vista conceptual como por lo que respecta a las técnicas correspondientes. O la insuficiencia de las técnicas tradicionales de evaluación de proyectos de inversión cuando se trata de proyectos de envergadura, que requieren una financiación compleja (Vélez-Pareja, 2007). O el tratamiento esquemático, si lo hay, de los problemas de diseño y asignación de tareas en líneas de producción o de montaje.

Pero, como ya se ha indicado, no se trata aquí de plantear una propuesta alternativa al paradigma vigente, sino de ilustrar con ejemplos la necesidad de una reflexión sobre el cambio.

\section{Conclusiones}

Medio siglo después de que se generara un nuevo sistema conceptual para la toma de decisiones en el área de operaciones, se impone una reflexión para renovar los contenidos de la enseñanza en este ámbito. Se trata de una tarea de gran magnitud, que sólo puede abordarse colectivamente.

En este trabajo se enumeran los cambios en el entorno que se han considerado más significativos en relación con la OP/DO y se analizan críticamente, teniendo en cuenta dichos cambios, los enfoques tradicionalmente adoptados para un cierto número de temas importantes del ámbito OP/DO. Asimismo, se formulan algunas propuestas orientadas a la renovación de los contenidos, con grados de detalle diversos.

El debate debería desembocar en un nuevo paradigma para la docencia de la OP/DO, que debería tener la repercusión correspondiente en la investigación. Las universidades, en los procedimientos que definan para el acceso a plazas de profesorado y los miembros de las comisiones correspondientes deberían propiciar la presentación de proyectos docentes críticos e innovadores.

\section{Referencias}

ALFALLA, R. y DOMÍNGUEZ. J.A. (2002) La formación en dirección de producción y operaciones. El caso de la Universidad Española. Secretariado de Publicaciones de la Universidad de Sevilla.

ALFORD, L.P. y BANGS, J.R. (1944). Production Handbook. The Ronald Press Co., New York.

BALINSKI, M. L. (1965). «Integer programming: methods, uses, computation». Management Science, |2, 253-3।3

BAÑEGIL, T.M. y RUBIO, S. (2005). «Sistemas de logística inversa en la empresa». Dirección y Organización, 31 , I I 0 116.

BIXBY, R.E. (2002). «Solving real-world linear problems: a decade and more of progress». Operations Research, 50, 3- 15.

BOITEUX, O.D.; COROMINAS, A. y LUSA, A. (2007). «Estado del arte sobre planificación agregada de la producción». Working Paper, Universitat Politècnica de Catalunya (http://hdl.handle.net/2 1 17/747)

BOWMAN, R. A. (2007) «Efficient sensitivity analysis of PERT network performance measures to significant changes in activity time parameters». Journal of the Operational Research Society, 58, I 354-1360.

BUFFA, E. S. ( 196 I) Modern production management. J.Wiley \& Sons, New York.

CHASE, R. B.; AQUILANO, N. J. y JACOBS, F.R. (2005). Administración de producción y operaciones ( $10^{\mathrm{a}}$ ed.). McGraw-Hill.

CHOPRA, S.; LOVEJOY,W. y YANO, C. (2004). «Five decades of operations management and the prospects ahead». Management Science, 50, 8- I4.

DAVIS, M. (197I) «That's Interesting!: Towards a Phenomenology of Sociology and a Sociology of Phenomenology», Philosophy of the Social Sciences, I, 309-344.

FORRESTER, J.W. (196I). Industrial Dynamics. MIT Press, Cambridge.

GAO, J.; GEN, M.; SUN, L. ZHAO, X. (2007). «A hybrid of genetic algorithm and bottleneck shifting for multiobjective flexible job shop scheduling problems». Computers \& Industrial Engineering, 53, 149-162.

HARRIS, F.W. (1913). «How many parts to make at once». Factory, The Magazine of Management, I0, I 35- I 36, 152. Reimpreso en Operations Research, 1990, 38, 6, 947-950.

HAYES, R. H. (2008) «Operations Management's next source of galvanizing energy?». Production and Operations Management, 17, 567-572.

HEIZER, J. y RENDER, B. (2007a). Dirección de la Producción. Decisiones estratégicas ( $8^{\mathrm{a}}$ ed.). Prentice-Hall. 
HEIZER, J. y RENDER, B. (2007b). Dirección de la Producción. Decisiones tácticas ( $8^{a}$ ed.). Prentice-Hall.

HOLT, C.C.; MODIGLIANI, F.y MUTH, J.F. (1956). «Derivation of a linear decision rule for production and employment scheduling》. Management Science, 2, I59- 177.

HOLT, C.C.; MODIGLIANI, F. y SIMON, H.A. (I 955). «A linear decision rule for production and employment scheduling». Management Science, 2, I-30.

HOPP,W.J. (2004). «Fifty years of Management Science». Management Science, 50, I-7.

JOHNSON S. M. (1954). «Optimal two- and three-stage production schedule with setup times included». Naval Research Logistics, I, 61-68.

KARMARKAR, U. (2004) «Will you survive the services revolution?». Harvard Business Review, 82, 6, 100-107.

KUO, C.-C. y WHITE, R.E. (2004) «A note on the treatment of the Center-of-Gravity Method in Operations Management textbooks». Decision Sciences Journal of innovative education, 2, 2, 219-227.

LEE, H. L., PADMANABHAN,V.y WHANG, S. (1997a) «lnformation distortion in a supply chain:The bullwhip effect». Management Science, 43 (4) 546-558.

LEE, H. L., PADMANABHAN, V. y WHANG, S. ( $997 b$ ) «The bullwhip effect in supply chain». Sloan Management Review, 38 (3), 93- 102.

MACHUCA, J.A.D.; GONZÁLEZ-ZAMORA, M.M. y AGUILAR-ESCOBAR, V.G. (2007) «Service Operations Management research». Journal of Operations Management, 25, 585-603.

MIRANDA, F.J.; RUBIO, S.; CHAMORRO, A. y BAÑEGIL,T. (2005). Manual de dirección de operaciones. Thomson.

MOHAN, S.; GOPALAKRISHNAN, M.; BALASUBRAMANIAN, H. y CHANDRASHEKAR, A. (2007) «A lognormal approximation of activity duration in PERT using two time estimates». Journal of the Operational Research Society, 58, $827-831$.

MONDEN, Y. ( 1 983). The Toyota Production System. Industrial Engineering and Management Press, Atlanta.
RUBIO, S., CHAMORRO, A. y F.J. MIRANDA. (2008). «Characteristics of the research on reverse logistics». International Journal of Production Research, 46 (4), I099- I 120.

SAMPSON, S. E. y FROEHLE, C. M. (2006). « Foundations and implications of a proposed Unified Services Theo-ry»». Production and Operations Management, I5, 329-343.

SINGHAL, K.; SINGHAL, J.y STARR, M.K. (2007). «The domain of production and operations management and the role of Elwood Buffa in its delineation». Journal of Operations Management, 25, 31 0-327.

SINGHAL, K. y SINGHAL, J. (2007). «Holt, Modigliani, Muth, and Simon's work and its role in the renaissance and evolution of operations management». Journal of Operations Management, 25, 300-309.

STIGLER, G.J. ( 1978). "The literature of Economics: the case of the kinked oligopoly demand curve." Economic Inquiry, 16, 185-204. Reimpreso en Stigler, G.C. (1982) The Economist as a preacher and other essays. The University of Chicago. (Edición española: «La literatura de la Economía: el caso de la curva de demanda quebrada del oligopolio», en El economista como predicadory otros ensayos, Folio, 1987, 334-365).

SMITH, J.S.; KARWAN, K.R. y MARKLAND, R.E. (2007) «A note on the growth of research in Service Operations Management». Production and Operations Management, 16, 6, 780-780.

TAJ, S.; HORMOZI, A.M. y MIRSHAB, B. (1996). «Undergraduate academic teaching and manufacturing industry requirement: a comparative analysis». Interfaces, 26, 5 I-57.

URWICK, L.; BRECHT, E.F.L. ( 1984$)$ La historia del management. Orbis, Barcelona.

VÉLEZ-PAREJA, I. (2007) «Cash-flow valuation in an inflationary world. The case of World Bank for regulated firms». Economic Analysis Working Papers 18 pp. (www.doaj.org).

WAGNER H.M. y WHITIN T.M.. (1958) «A dynamic version of the economic lot size model». Management Science, 5, 1, 89-96.

WOMACK, J.P.; JONES, D.T. y ROOS, D. (1990). The machine that changed the world. Rawson Associates. 